\title{
Streptococcus bovis
}

National Cancer Institute

\section{Source}

National Cancer Institute. Streptococcus bovis. NCI Thesaurus. Code C86784.

A species of facultatively anaerobic, Gram positive, cocci shaped bacteria in the phylum Firmicutes. This species is positive for arginine deamination and negative for catalase, oxidase, hemolysis, growth in $6.5 \% \mathrm{NaCl}$ and urease. It can ferment raffinose but not arabinose, mannitol, lactose or glycerol. S. bovis is found in the alimentary tract of ruminants and is a human pathogen that can cause bacteremia, sepsis or endocarditis. 